\title{
Reliability of response spike timings in pulse-coupled networks of neurons
}

\author{
Jun-nosuke Teramae* and Tomoki Fukai
}

\author{
Address: Brain Science Institute, RIKEN, Wako, Saitama, 351-0198, Japan \\ Email: Jun-nosuke Teramae* - teramae@riken.jp \\ * Corresponding author
}

from Eighteenth Annual Computational Neuroscience Meeting: CNS*2009

Berlin, Germany. 18-23 July 2009

Published: 13 July 2009

BMC Neuroscience 2009, I0(SuppI I):P93 doi:I0.I I86/I47I-2202-I0-SI-P93

This abstract is available from: http://www.biomedcentral.com/I47I-2202/I0/SI/P93

(c) 2009 Teramae and Fukai; licensee BioMed Central Ltd.

\section{Introduction}

Reliable information processing requires a code that can be represented and transmitted reliably within the precision of the devices. In the brain, neurons use spikes to convey information to other neurons and temporal codes hypothesize that information is encoded into fine spatiotemporal structure of precisely timed spike trains. Single neurons are known to generate highly precise spike trains when they are repeatedly activated by the same fluctuating input [1]. Neurons, however, work collectively rather than individually in their network. It remains unclear whether neurons in a network can still respond precisely since mutual couplings may affect the response of the individual neurons. To answer this question, we investigate the temporal precision of responses of a pulse-coupled network of neurons when a set of independent fluctuating inputs, frozen noise, is repeatedly applied to the network.

\section{Methods}

To study this problem analytically, we introduce uncoupled copies of the network that commonly receive the set of fluctuating inputs. Suppose that the original network repeatedly generates identical precisely timed spike responses. Then, we can interpret these responses across trials as responses of the individual copies in a trial. Thus, in-phase synchronization between identical networks implies precisely timed responses of a single network across trials. Such noise-induced synchronization was previously studied between single oscillators [2]. Here, we study the noise-induced synchronization between networks of neurons to clarify whether each network is able to encode information about fluctuating inputs into precisely timed spikes [3].

\section{Results}

We develop a mean-field theory of noise-induced synchronization between the copies of the pulse-coupled network of neurons. We can analytically derive a selfconsistent equation for the distribution of spike-time differences between the corresponding neurons and obtain the distribution as a function of the coupling strength of the network, which allows us to reveal the nontrivial effect of mutual couplings on the temporal precision of response spikes in the recurrent network of neurons. Spike sequence generated by the network is not perfectly the same across trials. Instead, the result implies enough coherence of spike trains between different trials, as indicated by the obvious peak of the distribution. The width of the peak measures a degree of the coherence, up to which spike trains can be used reliably. The result tells us how the coherence changes qualitatively as a function of the coupling strength in the network.

\section{Acknowledgements}

This work was supported by Grant-in-Aid for Young Scientists (B) 20700304. 


\section{References}

I. Mainen ZF, Sejnowski TJ: Reliability of spike timing in neocortical neurons. Science 1995, 268:1503-1506.

2. Teramae J, Tanaka D: Robustness of the noise-induced phase synchronization in a general class of limit cycle oscillators. Phys rev Lett 2004, 93:204103- I-4.

3. Teramae J, Fukai T: Temporal precision of spike response to fluctuating input in pulse-coupled networks of oscillating neurons. Phys Rev Lett 2008, 10 I:248105- 1-4.

Publish with Biomed Central and every scientist can read your work free of charge

"BioMed Central will be the most significant development for disseminating the results of biomedical research in our lifetime. " Sir Paul Nurse, Cancer Research UK

Your research papers will be:

- available free of charge to the entire biomedical community

- peer reviewed and published immediately upon acceptance

- cited in PubMed and archived on PubMed Central

- yours - you keep the copyright

Submit your manuscript here:

http://www.biomedcentral.com/info/publishing_adv.asp 